OPEN ACCESS

Edited by:

George C. Tsokos, Harvard Medical School,

United States

Reviewed by: Fabio Malavasi,

Università degli Studi di Torino, Italy

Caroline Jefferies,

Cedars-Sinai Medical Center,

United States

*Correspondence:

Jason S. Knight

jsknight@umich.edu

Specialty section:

This article was submitted

to Inflammation,

a section of the journal

Frontiers in Immunology

Received: 21 December 2017

Accepted: 28 May 2018

Published: 11 June 2018

Citation:

Knight JS, Mazza LF, Yalavarthi S,

Sule G, Ali RA, Hodgin JB, Kanthi Y and Pinsky DJ (2018)

Ectonucleotidase-Mediated

Suppression of Lupus Autoimmunity and Vascular Dysfunction.

Front. Immunol. 9:1322.

doi: 10.3389/fimmu.2018.01322

\section{Ectonucleotidase-Mediated Suppression of Lupus Autoimmunity and Vascular Dysfunction}

\author{
Jason S. Knight ${ }^{1 *}$, Levi F. Mazza', Srilakshmi Yalavarthi ${ }^{1}$, Gautam Sule ${ }^{1}$, Ramadan A. Ali ${ }^{1}$, \\ Jeffrey B. Hodgin ${ }^{2}$, Yogendra Kanthi ${ }^{3,4}$ and David J. Pinsky ${ }^{4,5}$ \\ 'Division of Rheumatology, Department of Internal Medicine, University of Michigan, Ann Arbor, MI, United States, \\ ${ }^{2}$ Department of Pathology, University of Michigan, Ann Arbor, MI, United States, ${ }^{3}$ Division of Cardiology, Ann Arbor \\ Veterans Administration Healthcare System, Ann Arbor, MI, United States, ${ }^{4}$ Division of Cardiovascular Medicine, \\ Department of Internal Medicine, University of Michigan, Ann Arbor, MI, United States, ${ }^{5}$ Department of Molecular \\ and Integrative Physiology, University of Michigan, Ann Arbor, MI, United States
}

Objectives: CD39 and CD73 are surface enzymes that jut into the extracellular space where they mediate the step-wise phosphohydrolysis of the autocrine and paracrine danger signals ATP and ADP into anti-inflammatory adenosine. Given the role of vascular and immune cells' "purinergic halo" in maintaining homeostasis, we hypothesized that the ectonucleotidases CD39 and CD73 might play a protective role in lupus.

Methods: Lupus was modeled by intraperitoneal administration of pristane to three groups of mice: wild-type (WT), CD39-/-, and CD73--. After 36 weeks, autoantibodies, endothelial function, kidney disease, splenocyte activation/polarization, and neutrophil activation were characterized.

Results: As compared with WT mice, CD39-/- mice developed exaggerated splenomegaly in response to pristane, while both groups of ectonucleotidase-deficient mice demonstrated heightened anti-ribonucleoprotein production. The administration of pristane to WT mice triggered only subtle dysfunction of the arterial endothelium; however, both $\mathrm{CD} 39^{-/-}$and $\mathrm{CD} 73^{-/-}$mice demonstrated striking endothelial dysfunction following induction of lupus, which could be reversed by superoxide dismutase. Activated B cells and plasma cells were expanded in $\mathrm{CD} 73^{-/}$mice, while deficiency of either ectonucleotidase led to expansion of $\mathrm{T}_{H} 17$ cells. CD39-/- and $\mathrm{CD} 73^{-/-}$mice demonstrated exaggerated neutrophil extracellular trap release, while $\mathrm{CD} 73^{-/-}$mice additionally had higher levels of plasma cell-free DNA.

Conclusion: These data are the first to link ectonucleotidases with lupus autoimmunity and vascular disease. New therapeutic strategies may harness purinergic nucleotide dissipation or signaling to limit the damage inflicted upon organs and blood vessels by lupus.

Keywords: systemic lupus erythematosus, ectonucleotidases, CD73, CD39, $T_{H} 17$ cells, endothelial dysfunction, neutrophil extracellular traps

\section{INTRODUCTION}

Systemic lupus erythematosus (commonly referred to as "lupus") is the prototypical systemic autoimmune disease. In the United States, the prevalence of lupus approaches 1 in 500, with a disproportionate impact on women of childbearing age and minorities. The immunopathology of lupus is complex, with derangements present in both lymphocyte- and myeloid-lineage cells. 
Beyond the well-recognized damage inflicted by lupus upon organs such as kidneys, skin, and joints, lupus cardiovascular disease has emerged as a leading cause of morbidity and mortality. Indeed, young women with lupus carry a 50-fold increased risk of cardiovascular events when compared with their unaffected peers (1).

Although the proximal trigger for lupus is unknown, there is some evidence that environmental factors are contributory. In mice, the intraperitoneal administration of pristane (a naturally occurring hydrocarbon) recruits inflammatory macrophages into the peritoneal cavity, where they robustly produce type I interferons $(2,3)$. Mediated at least in part by these interferons, female mice take on features of lupus over the ensuing 6-9 months, including anti-ribonucleoprotein (anti-RNP) antibody production, splenic immune cell derangements, and immune complex glomerulonephritis $(2,3)$. Mechanistically, the pristane model of lupus depends upon both the type I interferon receptor and tolllike receptor 7 for autoantibody formation and other aspects of the lupus phenotype $(4,5)$. In recent years, the pristane model has been used to assess wide-ranging concepts in lupus pathogenesis including the roles of leptin (6), selectin-mediated leukocyte adhesion (7), and the inflammasome (8).

Leukocytes and endothelial cells are regulated by a dynamic halo of ATP, ADP, AMP, and adenosine. Purine nucleotides are liberated in large quantities from dying cells at sites of hypoxic, ischemic, or inflammatory stress (9). ATP and ADP then engage cell-surface receptors to launch proinflammatory and prothrombotic cascades (9). By contrast, adenosine (the extracellular concentration of which can rise by orders of magnitude during acute inflammation) has potent antithrombotic, anti-inflammatory, and immunosuppressive properties mediated by surface $G$ proteincoupled receptors (10).

To regulate the local concentrations of purine nucleotides and adenosine, the ectonucleotidases CD39 and CD73 extend into the extracellular space from the surfaces of leukocytes and endothelial cells (Figure 1A). CD39 is a membrane-spanning enzyme with an ectodomain that cleaves the terminal phosphate group from ATP to form ADP, and then from ADP to form AMP (11). From there, CD73 (a GPI-anchored protein) clips the final phosphate group from AMP to generate adenosine (11). The endothelium is a key site of ectonucleotidase expression, with well-recognized upregulation in response to stressors such as hypoxia, thereby limiting leukocyte activity and efflux (12-14). Leukocytes (including lymphocytes and neutrophils) also express ectonucleotidases, not only to autoregulate activation, adhesion, and transit but also to manipulate neighboring cells $(15,16)$. Indeed, specialized immune cells such as regulatory $\mathrm{T}$ cells (Tregs) and myeloid-derived suppressor cells mediate their effects in part through local ectonucleotidase-generated adenosine $(15,16)$. To the best of our knowledge, the only connection between ectonucleotidases and lupus reported to date is the observation that some lupus patients lack adequate T-cell expression of CD39 and CD73, hinting at a defect in regulatory T-cell function $(17,18)$. The studies described here seek to provide new insight into how a pathway that functions as an endogenous guardian against inflammation may be exploited to counteract lupus.
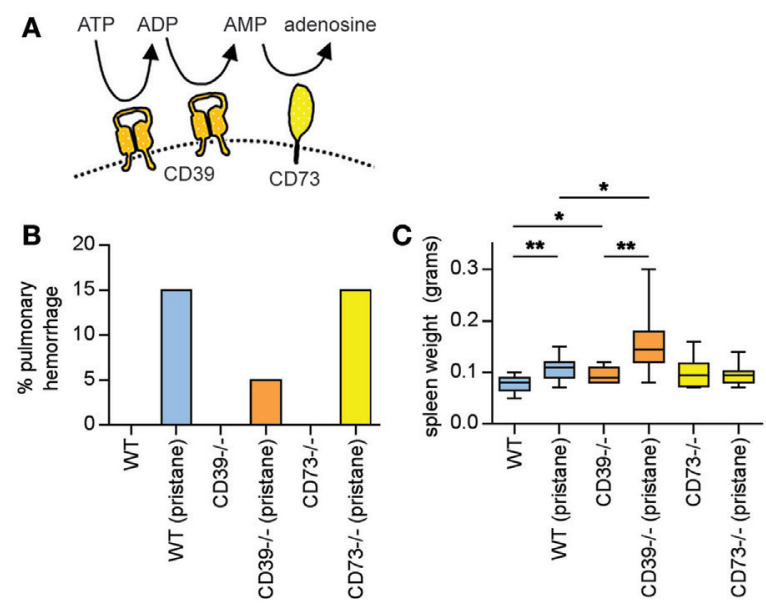

FIGURE 1 | Pulmonary hemorrhage and splenomegaly in pristane-treated mice. (A) Schematic of CD39 and CD73, which together mediate the step-wise phosphohydrolysis of extracellular ATP into adenosine. (B) Some mice developed clinically overt pulmonary hemorrhage within the first month after pristane administration; these mice required euthanasia. $N=10$ saline-treated mice and 20 pristane-treated mice per genotype; no comparisons were statistically significant. (C) Mice were administered either saline or pristane, as indicated. 36 weeks later, spleen size was measured. $N=10$ per control group and 17-19 per pristane group; ${ }^{\star} p<0.05$ and ${ }^{* *} p<0.01$. Box-and-whisker plots denote minimum, 25th percentile, median, 75th percentile, and maximum.

\section{MATERIALS AND METHODS}

\section{Animal Housing and Treatments}

Mice were housed in a specific pathogen-free barrier facility, and fed standard chow. Female C57BL/6 mice were purchased from The Jackson Laboratory. CD $39^{-/-}$mice have been described by our group previously (19). CD73-- mice in the C57BL/6 background were originally obtained from Dr. Linda Thompson and have been used by our group previously (20). Pristane was purchased from Sigma. At 8-10 weeks of age, female mice were administered a single intraperitoneal dose of $500 \mu \mathrm{l}$ pristane or $500 \mu \mathrm{l}$ normal saline. Unless otherwise indicated, studies were performed on mice euthanized at 36 weeks. This study was carried out in accordance with the recommendations of the National Research Council, Guide for the Care and Use of Laboratory Animals. The protocol was approved by the Institutional Animal Care and Use Committee.

\section{Complete Blood Counts}

Peripheral leukocyte and platelet counts were determined with an automated Hemavet 950 counter (Drew Scientific).

\section{ELISAs}

Kits for mouse anti-nRNP IgG (5415) and mouse total IgG (6320) were purchased from Alpha Diagnostic International and performed according to the manufacturer's instructions.

\section{Kidney Scoring}

At the time of euthanasia, kidneys were gently perfused with heparinized saline. A portion of the cortex was frozen in Tissue-Tek 
OCT (Sakura Finetek) for immunofluorescence staining. Staining for kidney IgG and C3 was performed on frozen sections as described $(21,22)$. Another portion of the cortex was fixed in formalin and embedded in paraffin. Formalin-fixed sections were stained by periodic acid-Schiff and then scored in a blinded manner as previously described $(21,22)$. In brief, a semiquantitative scoring system: 0 , no involvement; 0.5 , minimal involvement of $<10 \%$; 1 , mild involvement (10-30\% section); 2 , moderate involvement (31-60\% of section); and 3, severe involvement. This system was used to assess 13 different parameters of activity and chronicity (mesangial hypercellularity, mesangial deposits, mesangial sclerosis, endocapillary cellular infiltrate, subepithelial and subendothelial deposits, capillary thrombi, capillary sclerosis, cellular or organized crescents, synechiae, tubular atrophy, and interstitial fibrosis). For glomerular indices, 30 glomeruli were examined per mouse, and a cumulative score was determined for each parameter.

\section{Flow Cytometry}

A single-cell suspension of splenocytes was analyzed with the following anti-mouse antibodies (all from BioLegend): CD138, B220, CD80, CD19, CD44, and CD62L. Mouse Th1/Th17 Phenotyping Kit was from BD Pharmingen and performed according to the manufacturer's instructions. Staining was typically for $30 \mathrm{~min}$ at $4^{\circ} \mathrm{C}$. After washing, cells were fixed in $2 \%$ paraformaldehyde before analysis with a CyAn ADP Analyzer (Beckman Coulter). Further data analysis was done using FlowJo analysis software.

\section{Endothelial Function}

Studies were performed as previously reported by our group $(21,22)$. After euthanasia with pentobarbital, thoracic aortas were excised, cleaned, and cut into $2-\mathrm{mm}$ length rings. The endothelium was left intact and rings were mounted in a myograph system (Danish Myo Technology A/S). Rings were bathed with warmed and aerated $\left(95 \% \mathrm{O}_{2} / 5 \% \mathrm{CO}_{2}\right)$ physiological salt solution. Aortic rings were set at $7 \mathrm{mN}$ passive tension and equilibrated for $1 \mathrm{~h}$. Cumulative doses of phenylephrine $\left(10^{-9}\right.$ to $\left.10^{-6} \mathrm{M}\right)$ were then added to the bath to establish a concentration-response curve. After washing, a phenylephrine concentration corresponding to $80 \%$ of the maximum was added, and contraction was allowed to reach a stable plateau. To examine endothelium-dependent relaxation, acetylcholine $\left(10^{-9}\right.$ to $\left.10^{-6} \mathrm{M}\right)$ was added cumulatively to the bath. Finally, a normal vascular smooth muscle response was confirmed by washing out phenylephrine and acetylcholine, and then repeating the experiment with phenylephrine contraction followed by cumulative addition of sodium nitroprusside $\left(10^{-9}\right.$ to $\left.10^{-5} \mathrm{~mol} / \mathrm{L}\right)$. In some experiments, one aortic ring from each mouse was treated as usual, while a second ring was incubated with superoxide dismutase (SOD) $1.2 \mathrm{kU} / \mathrm{ml}$ during the equilibration phase of the experiment.

\section{Blood Pressure}

Non-invasive blood pressure was measured by tail cuff as described (23). Briefly, using the IITC Life Science blood pressure measurement system, conscious and restrained mice were acclimated for 3 days in a temperature controlled environment (model 306 warming chamber). The tail vein was occluded with an integrated sensor-cuff (model I-B60-1/4) and return of pulsation (RTP) detected by the RTP-computerized blood pressure monitor (model 6M 2296 channel mouse system). Repeated measures were averaged for determination and report of systolic blood pressure and heart rate.

\section{Quantitative PCR}

At the time of tissue harvest, aortas were snap frozen in liquid nitrogen and stored at $-80^{\circ} \mathrm{C}$. Later, the aortas were mechanically homogenized in TriPure Isolation Reagent (Roche). RNA was prepared by the Direct-zol RNA MiniPrep kit (Zymo Research) according to the manufacturer's instructions. RNA integrity number was $>7$ for all included samples. cDNA was synthesized using MMLV RT (Invitrogen) and 100 ng of RNA using a MyCycler thermocyler (Bio-Rad). Quantitative PCR was with SYBR Green PCR Master Mix (Applied Biosystems) according to the manufacturer's instructions, and carried out using an ABI PRISM 7900HT (Applied Biosystems). Primers for the housekeeping gene beta-actin were purchased from Qiagen (QuantiTect Primer Assays, which have proprietary primer sequences). Primer sequences for endothelial nitric oxide synthase (eNOS) were 5'-GACCCTCACCGCTACAACAT-3' and 5'-TTTGGCCAGCTGGTAACTGT-3'; primer sequences for inducible NOS (iNOS) were $5^{\prime}$-TGGTGGTGACAAGCAC ATTT- $3^{\prime}$ and 5'-GCCAAACACAGCATACCTGAA-3'. Ct values were normalized to the housekeeping gene to determine $\Delta \mathrm{Ct}$. $\Delta \Delta \mathrm{Ct}$ values were then determined by comparing each $\Delta \mathrm{Ct}$ to the average $\Delta \mathrm{Ct}$ for the wild-type (WT) control group. Data were presented as relative fold change by the formula $2^{\Delta \Delta C t}$.

\section{Measurement of Cell-Free DNA}

Cell-free DNA was quantified in plasma using the Quant-iT PicoGreen dsDNA Assay Kit (Invitrogen) according to the manufacturer's instructions.

\section{Neutrophil Purification and NETosis Assay}

Bone marrow neutrophils were isolated as previously described $(21,22)$. Briefly, total bone marrow cells were spun on a discontinuous Percoll gradient $(52-69-78 \%)$ at $1,500 \times g$ for $30 \mathrm{~min}$. Cells from the $69-78 \%$ interface were collected. These cells were $>95 \%$ Ly-6G-positive by flow cytometry and had typical nuclear morphology by microscopy. To assess in vitro NETosis, a protocol similar to what we have described previously was followed $(21,22)$. Culture was for $4 \mathrm{~h}$ at $37^{\circ} \mathrm{C}$ in RPMI media supplemented with $2 \%$ bovine serum albumin and $10 \mathrm{mM}$ HEPES buffer. Stimulation with phorbol-12-myristate-13-acetate (100 nM, Sigma) was also for $4 \mathrm{~h}$. For immunofluorescence, cells were fixed with $4 \%$ paraformaldehyde. DNA was stained with Hoechst 33342 (Invitrogen), while protein staining was with rabbit polyclonal antibody to citrullinated histone $\mathrm{H} 3$ (Abcam), followed by FITC-conjugated anti-rabbit IgG (SouthernBiotech). Images were collected with an Olympus IX70 microscope and a CoolSNAP HQ2 monochrome camera (Photometrics) with Metamorph Premier software (Molecular Devices). Neutrophil extracellular traps (NETs) (decondensed areas of extracellular DNA co-staining with one of the aforementioned protein markers) were quantified by two blinded 
observers, and digitally recorded to prevent multiple counts; the percentage of NETs was calculated after counting $10400 \times$ fields per sample.

\section{Statistical Analysis}

Data analysis was with GraphPad Prism software version 6. For continuous variables, normally distributed data were analyzed by unpaired two-tailed $t$ testing, while skewed data were assessed by Mann-Whitney test. For dichotomous variables, analysis was by Chi square. For endothelial function experiments, curve fit was by the least squares method, and comparisons were by twoway ANOVA. Statistical significance was defined as $p<0.05$.

\section{RESULTS}

The one-time intraperitoneal administration of the natural hydrocarbon pristane promotes features of lupus, which emerge over 6-9 months $(2,3)$. Here, pristane was administered to three groups of mice at 8-10 weeks of age, all in the C57BL/ 6 background: WT, $\mathrm{CD} 39^{-/-}$, and $\mathrm{CD}_{3}{ }^{-1-}$. Twenty mice were treated with pristane and 10 with saline for each genotype.

A feature of pristane administration to $\mathrm{C} 57 \mathrm{BL} / 6$ mice is the induction of diffuse alveolar hemorrhage, which becomes clinically apparent in 15-20\% of mice approximately 4 weeks after treatment $(24,25)$. Mechanistically, roles have been suggested for both B cells (24) and macrophages (25) in the pulmonaryhemorrhage phenotype. Here, three WT mice (15\%), one CD39--mouse $(5 \%)$, and three $\mathrm{CD}^{-/-}$mice $(15 \%)$ developed clinically overt pulmonary hemorrhage in response to pristane (and required euthanasia), all within 4 weeks of pristane administration (Figure 1B). No saline-treated mouse developed a similar phenotype (Figure 1B). All remaining mice survived to 36 weeks, at which time they were euthanized.

\section{CD39 Deficiency Potentiates Splenomegaly in Response to Pristane}

At 36 weeks, pristane promoted splenomegaly in WT mice, which was further potentiated by CD39 deficiency (Figure 1C).

\section{Ectonucleotidase Deficiency Potentiates Autoimmunity in Pristane-Treated Mice}

Pristane administration induces the production of autoantibodies, especially to ribonucleoproteins (RNPs) $(2,3)$. Here, serum anti-RNP antibody levels were measured 36 weeks after pristane administration. While WT mice demonstrated a strong trend toward induction of anti-RNP antibodies with pristane administration $(p=0.054)$, this induction was further potentiated by ectonucleotidase deficiency (Figure 2A). Anti-RNP antibodies were not detected at a significant level in any of the saline-treated groups (Figure 2A). We also measured serum total IgG. In WT mice, pristane triggered higher levels of IgG when compared with saline-treated controls (Figure 2B). A trend for increased IgG was also observed in the ectonucleotidase-deficient mice, although this did not reach statistical significance (which may have been at least partially attributable to a higher "baseline" in the ectonucleotidase-deficient controls) (Figure 2B). In summary,

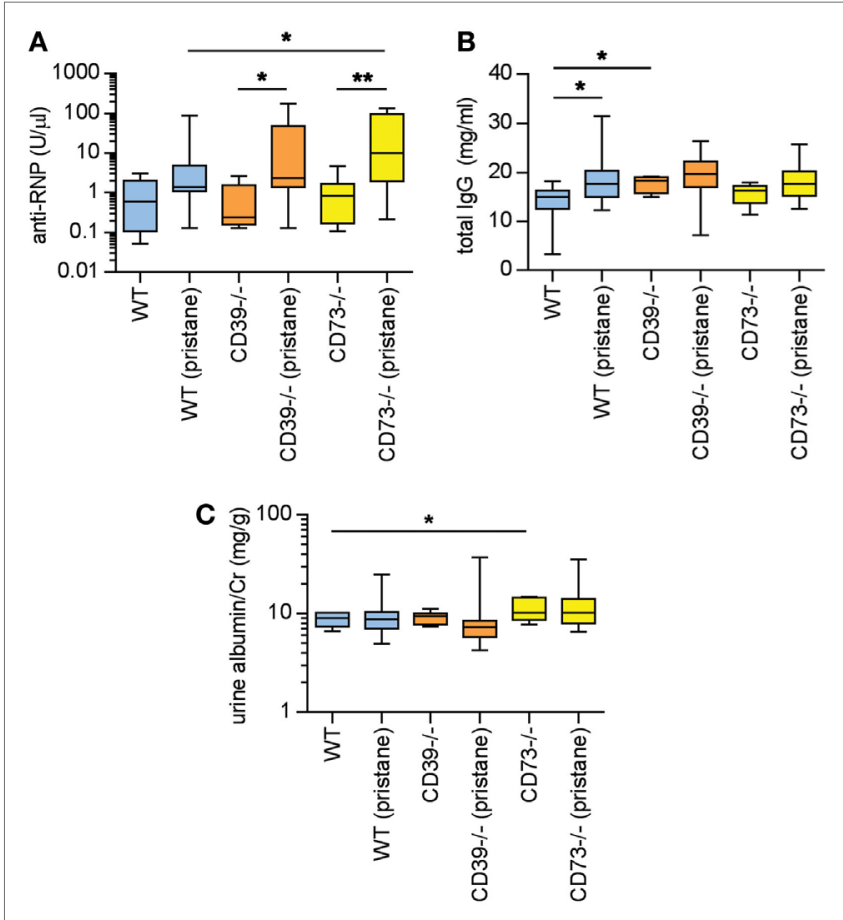

FIGURE 2 | Ectonucleotidase deficiency potentiates autoimmunity in pristane-treated mice. Mice were administered either saline or pristane, as indicated. 36 weeks later, various endpoints were tested. (A) Antiribonucleoprotein (Anti-RNP) IgG was measured in serum. (B) Total lgG was measured in serum. (C) Spot albumin/Cr (albumin/creatinine) ratios in urine. $N=10$ per control group and $17-19$ per pristane group. ${ }^{*} p<0.05$ and ${ }^{* *} p<0.01$

these data indicate that ectonucleotidase deficiency potentiates autoantibody formation, but not total IgG levels, in response to pristane.

\section{C57BL/6 Mice Do Not Develop Significant Proteinuria in Response to Pristane}

In previous studies of pristane administration to C57BL/6 mice, the kidneys have demonstrated a relatively mild phenotype of increased mesangial cellularity, which is compatible with World Health Organization Class II lupus nephritis in patients $(2,26)$. Immune complex and complement deposition have also been appreciated in pristane-treated C57BL/6 mice, albeit in the setting of minimal proteinuria $(2,26)$. Here, the albuminuria detected at 36 weeks was at a level that would be considered microalbuminuria (i.e., albumin/creatinine $<300 \mathrm{mg} / \mathrm{g}$ ) (Figure 2C); the only statistically significant difference between groups was that saline-treated $\mathrm{CD}^{-/-}$mice demonstrated higher levels of microalbuminuria than saline-treated WT mice. Beyond albuminuria, kidney glomeruli were scored for IgG and C3 deposition at 36 weeks. Pristane administration did enhance both IgG and C3 deposition when compared with saline-treated controls (Figures S1A-C in Supplementary Material); however, ectonucleotidase deficiency did not potentiate either IgG or C3 deposition. Kidney histopathology was also assessed across a variety of inflammatory parameters (see Materials and Methods). As compared with 
saline-treated controls, WT mice demonstrated an increase in mesangial hypercellularity upon pristane administration (Figures S1D,E in Supplementary Material); there were no other statistically significant differences between the groups. In summary, there is evidence that pristane administration induces modest glomerular immune complex deposition and mesangial hypercellularity, albeit without significant proteinuria, and without potentiation by ectonucleotidase deficiency.

\section{Ectonucleotidase Deficiency Expands B- and T-Cell Populations}

As above, there is evidence of exacerbated autoimmunity (splenomegaly, increased anti-RNP antibodies) when ectonucleotidasedeficient mice are treated with pristane. To understand this mechanistically, we first characterized splenic B cell populations. As compared with WT mice, $\mathrm{CD} 73^{-1-}$ mice demonstrated expansion of plasma cells (Figures $3 \mathbf{A}, \mathbf{B}$ ) and $\mathrm{CD} 80^{+}$activated B cells (Figures 3C,D); a similar expansion was not seen in $\mathrm{CD}^{29^{-/}}$mice. Moreover, as compared with pristane-treated WT mice, there was an increase in splenic follicular and marginal zone B cells in pristane-treated $\mathrm{CD}_{73^{-/}}$mice (Figure S2 in Supplementary Material). We also characterized splenic T cells. As compared with pristane-treated WT mice, there was an expansion of effector/ memory $\mathrm{CD}^{+} \mathrm{T}$ cells $\left(\mathrm{CD} 44^{\mathrm{hi}} \mathrm{CD}{ }^{2} \mathrm{~L}^{\mathrm{lo}}\right.$ ) in pristane-treated $\mathrm{CD}^{2} 3^{-1}$ mice (Figure 4A); interestingly, this increase was also noted in the saline-treated $\mathrm{CD}^{-1-} 3^{-1}$ controls. While there was no difference in IFN $\gamma$-producing $\mathrm{T}_{\mathrm{H}} 1$ cells (Figure 4B), IL-17A-producing $\mathrm{T}_{\mathrm{H}} 17$ cells were significantly increased in both $\mathrm{CD}^{-1-}$ and $\mathrm{CD}^{-1} 3^{-/-}$mice (Figure 4C). The $\mathrm{T}_{\mathrm{H}} 17$ expansion was robust-present at baseline (i.e., the saline groups) and then further potentiated by pristane (Figure 4C). In summary, these data demonstrate that ectonucleotidase deficiency promotes expansion of $\mathrm{T}_{\mathrm{H}} 17$ cells, while plasma cells and activated $\mathrm{B}$ cells are expanded only in the CD73-deficient mice.

\section{Ectonucleotidases Protect Against Endothelial Dysfunction in Pristane- Treated Mice}

Dysfunction of the arterial endothelium (as defined by impaired flow-mediated dilation) is a harbinger of atherosclerosis in lupus

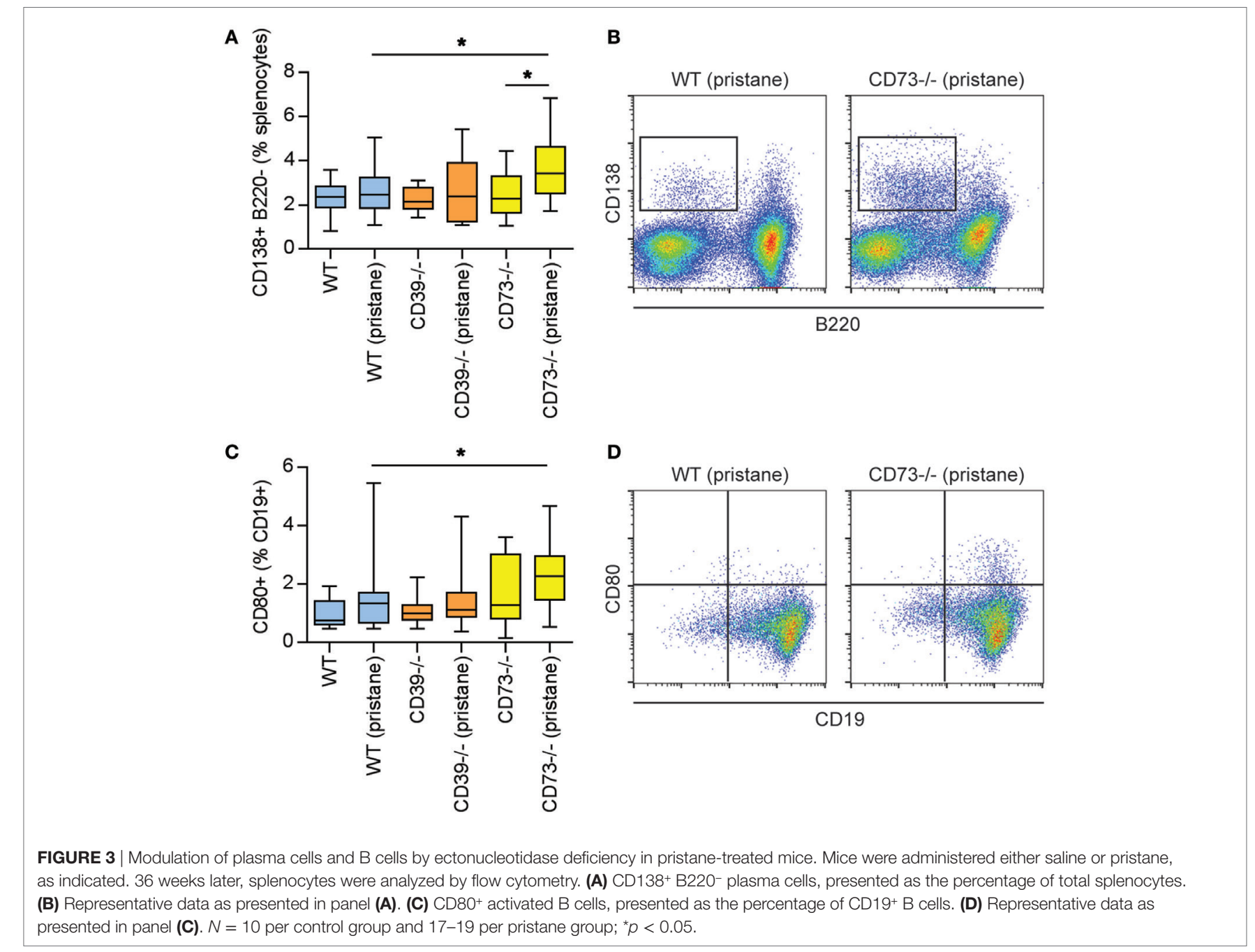



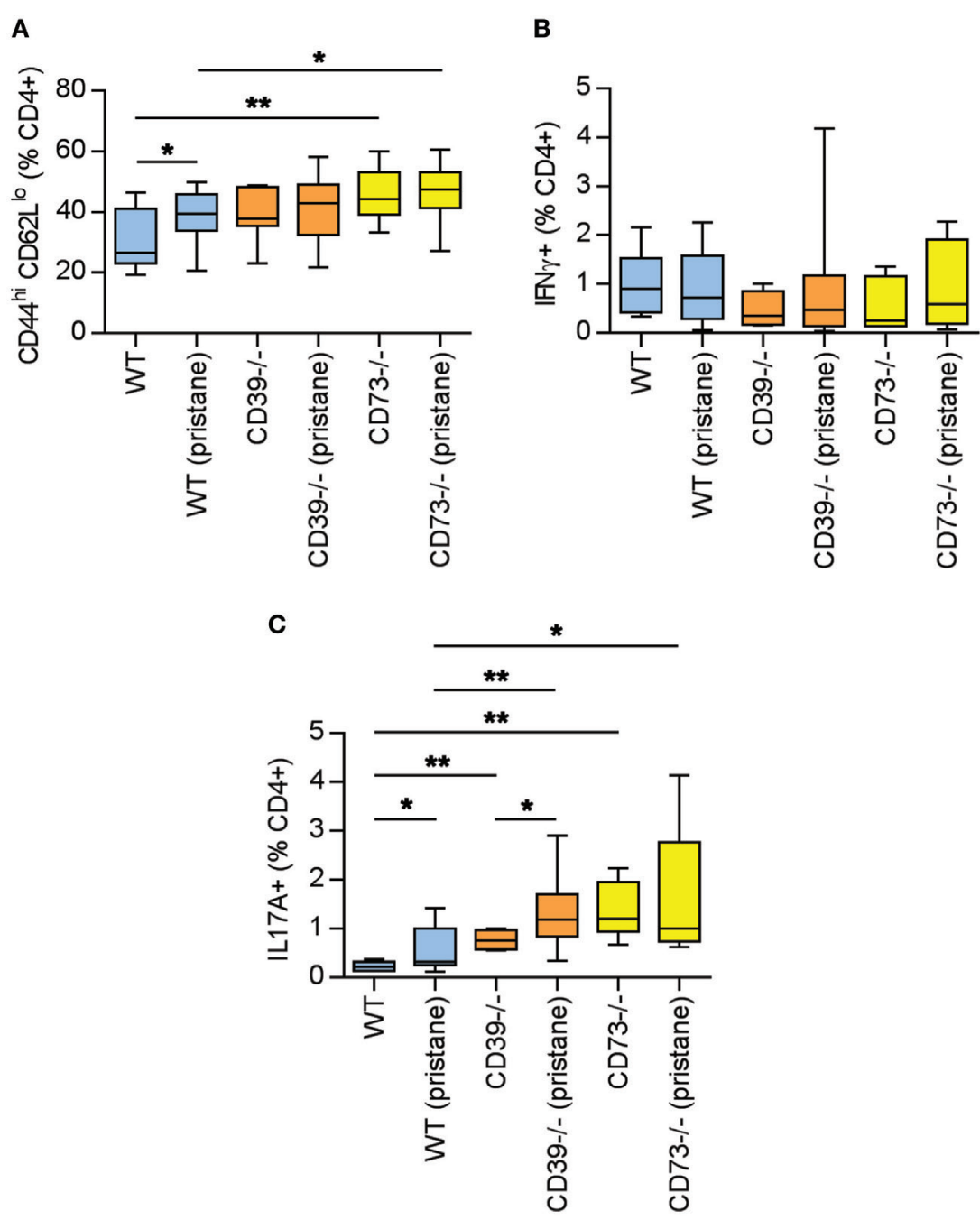

FIGURE 4 | Potentiation of $T$ cell activation and $T_{H} 17$ polarization by ectonucleotidase deficiency. Mice were administered either saline or pristane, as indicated. 36 weeks later, splenocytes were analyzed by flow cytometry. (A) CD44 ${ }^{\text {hi }}$ CD62 $L^{\text {lo }}$ effector/memory $T$ cells, presented as the percentage of CD4+ $T$ cells. (B) Percentage of CD4 ${ }^{+} \mathrm{T}$ cells expressing interferon gamma. (C) Percentage of CD4 ${ }^{+} \mathrm{T}$ cells expressing interleukin $17 \mathrm{~A}$. For panel (A), $n=10$ per control group and 17-19 per pristane group. For panels $\mathbf{( B , C ) , ~} n=5$ per control group and 8-10 per pristane group; ${ }^{*} p<0.05$ and ${ }^{* *} p<0.01$.

patients (27). Endothelial dysfunction is driven by oxidative stress, under which nitric oxide synthase becomes "uncoupled" and produces vasoconstrictive superoxide anion rather than vasodilatory nitric oxide (27). In both $\mathrm{CD} 39^{-/-}$and $\mathrm{CD}^{-/-}$mice (but not WT mice), pristane administration induced significant dysfunction of the arterial endothelium, when compared with their respective control groups (Figures 5A-C). Interestingly, $\mathrm{CD}_{39^{-1-}}$ mice demonstrated a trend toward more robust baseline aorta relaxation, when compared with the other two genotypes (Figures 5A-C). The endothelial dysfunction of $\mathrm{CD}^{-1-}$ and $\mathrm{CD} 3^{-/-}$mice was not explained by alterations in either blood pressure or heart rate (Figure S3 in Supplementary Material) and could be mitigated by ex vivo administration of SOD to aortic rings (to neutralize reactive oxygen species) (Figures 5D,E). We also assessed transcription of the genes for both eNOS and iNOS in aortas and found that eNOS transcription was upregulated in $\mathrm{CD} 39^{-/-}$and $\mathrm{CD} 73^{-/-}$mice when compared with WT mice, but was not further modified by pristane administration (Figure S4A in Supplementary Material). Transcription of iNOS was not significantly regulated by any of the conditions (Figure S4B in Supplementary Material). In summary, these data demonstrate that ectonucleotidases protect against dysfunction of the arterial endothelium in response to pristane, and that pristane-induced dysfunction can be rescued by administration of SOD.

\section{Ectonucleotidase Deficiency Potentiates Neutrophil Activation}

Neutrophils are being increasingly recognized as pathogenic agents in lupus $(28,29)$. For example, a "neutrophil signature" in blood heralds the onset of lupus nephritis in lupus patients $(30,31)$. NETs released by lupus neutrophils are at least one driver 

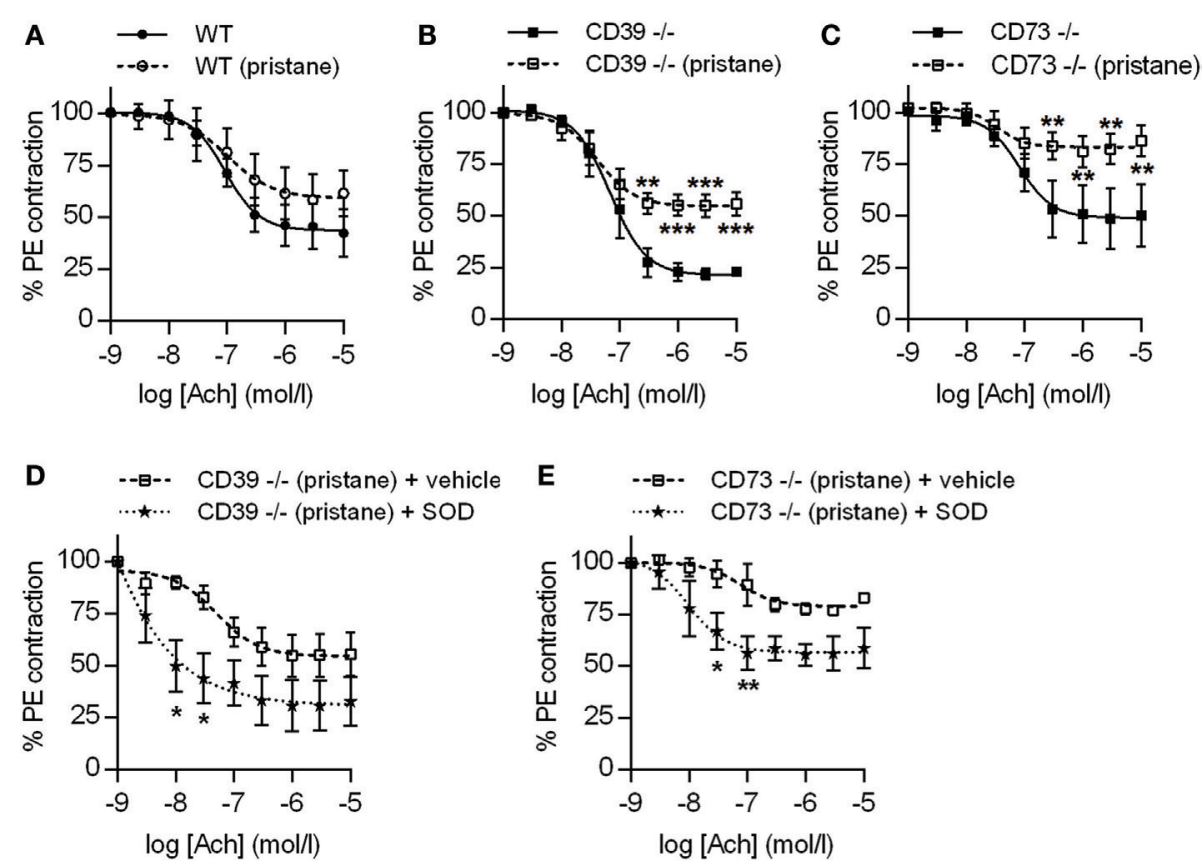

FIGURE 5 | Ectonucleotidase deficiency potentiates endothelial dysfunction in pristane-treated mice. Mice were administered either saline or pristane, as indicated. 36 weeks later, "aortic rings" were harvested for ex vivo determination of endothelial function by measuring the contractile force remaining in pre-contracted (by phenylephrine/PE) aortic rings in response to progressively increasing concentrations of acetylcholine. A "deeper" curve indicates a healthier endothelium, while a "flatter" curve denotes endothelial dysfunction. (A-C) $N=5$ control mice and 7 pristane mice per graph. (D,E) Two aortic rings were isolated from each mouse $(n=5)$, and one was treated with superoxide dismutase (SOD). ${ }^{*} p<0.05,{ }^{* *} p<0.01$, and ${ }^{* \star *} p<0.001$.

of type I interferon production (which counteracts endothelial homeostasis) $(32,33)$, while hyperactive neutrophils are likely an important stressor of the lupus endothelium $(21,22,34)$. As compared with WT mice, $\mathrm{CD}_{73^{-1}}$ mice demonstrated an increased neutrophil-to-lymphocyte ratio, especially in response to pristane (Figure 6A); the increased ratio was related to an increase in absolute neutrophil count, more so than a decrease in the lymphocyte count (Figure S5 in Supplementary Material). $\mathrm{CD} 3^{-1-}$ mice also demonstrated elevated levels of cell-free DNA (a surrogate for NETs) in serum (Figure 6B). Ex vivo, NET release was accelerated by deficiency of either CD39 or CD73, both at baseline (i.e., in the saline groups) and in response to pristane (Figures 6C,D). In summary, these data reveal that ectonucleotidases mitigate the release of NETs by neutrophils, both at baseline and in the context of lupus.

\section{DISCUSSION}

The literature already hints at an intersection between adenosine signaling and lupus. One study has suggested that lupus-prone Fas ${ }^{\mathrm{lpr} / \mathrm{pr}}$ mice are protected from nephritis by an adenosinereceptor agonist (35). In some lupus patients, there is increased adenosine deaminase activity (and presumably lower adenosine content) in blood $(36,37)$. Perhaps to compensate for this, adenosine-receptor density may be increased on lupus lymphocytes (38). Moreover, these pathways are potentially amenable to pharmacologic manipulation in patients. For example, adenosine signaling is indirectly amplified by a number of medications with relevance to rheumatology including methotrexate, dipyridamole, and phosphodiesterase 4 inhibitors.

Here, we have demonstrated for the first time a role for the ectonucleotidases CD39 and CD73 in protecting against lupus. Deletion of CD39 and CD73 leads to higher levels of anti-RNP antibodies in response to pristane, with CD73 deletion in particular promoting expansion of splenic $\mathrm{B}$ cell and $\mathrm{T}$ cell populations that likely contribute to autoantibody production. Within the $\mathrm{T}$ cell compartment, it is notable that some of these changes (expansion of effector/memory $\mathrm{T}$ cells and $\mathrm{T}_{\mathrm{H}} 17$ cells) were present independent of pristane administration, which would fit with a general predisposition toward inflammation and autoimmunity conferred by loss of ectonucleotidase activity $(11,39,40)$. For example, protective roles for ectonucleotidases have been suggested in rheumatoid arthritis $(41,42)$, juvenile idiopathic arthritis (43), inflammatory bowel disease $(44,45)$, autoimmune hepatitis (46), and atherosclerosis $(47,48)$. This is the first study to explore these pathways in lupus.

The classic markers of Tregs are CD25 and the forkhead transcription factor FoxP3. Recently, it has also been recognized that both CD39 and CD73 are surface markers of Tregs, generating adenosine to induce anergy in effector T cells via the $\mathrm{A}_{2 \mathrm{~A}}$ receptor $(15,49,50)$. In contrast to the protective role of Tregs, effector $T_{H} 17$ cells have a well-established role in promoting autoimmunity in various diseases including multiple sclerosis, rheumatoid arthritis, and lupus. Indeed, lupus mice and patients have increased frequency of circulating $\mathrm{T}_{\mathrm{H}} 17$ cells, which correlate with disease activity (51-53). Furthermore, the induction of lupus by pristane 

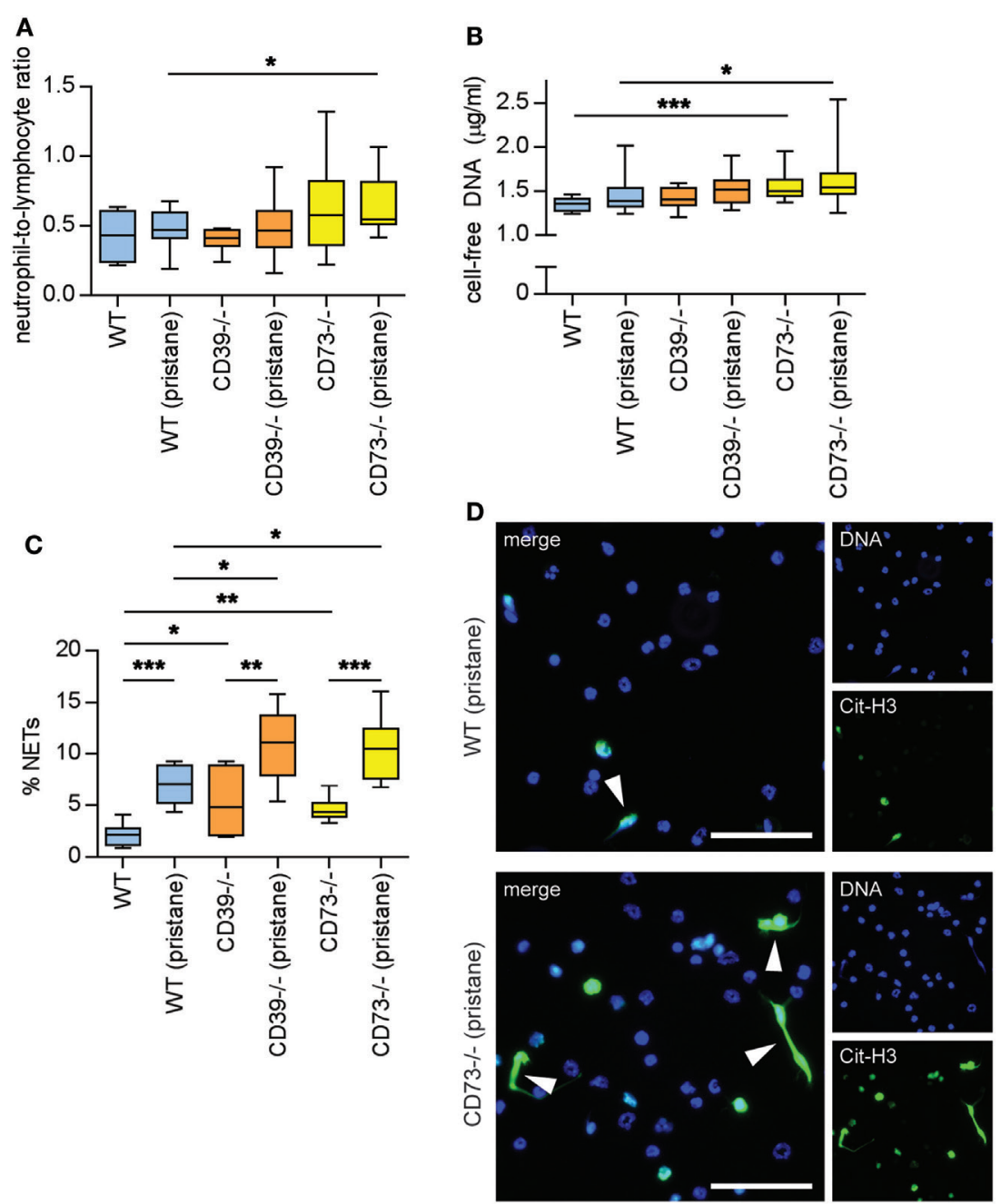

FIGURE 6 | Ectonucleotidase deficiency potentiates neutrophil activation. Mice were administered either saline or pristane, as indicated. 36 weeks later, various endpoints were assessed. (A) Neutrophil-to-lymphocyte ratio in peripheral blood. (B) Cell-free DNA in mouse serum. (C) Mature neutrophils were purified from mouse bone marrow and cultured for $4 \mathrm{~h}$ on polylysine-coated glass coverslips. Spontaneous neutrophil extracellular trap (NET) release was assessed by immunofluorescence microscopy. (D) Representative photomicrographs from the data presented in panel $\mathrm{C}$. DNA is stained blue and citrullinated histone $\mathrm{H} 3$ $(\mathrm{Cit}-\mathrm{H} 3)$ green. NETs are identified as extracellular areas of blue and green overlap (arrowheads). Scale bar $=50 \mu \mathrm{m}$. For panels $(\mathbf{A}, \mathbf{B}), n=10$ per control group and 17-19 per pristane group. For panel (C), $n=6-9$ per group; ${ }^{\star} p<0.05,{ }^{\star \star} p<0.01$, and ${ }^{\star \star *} p<0.001$.

is significantly mitigated by deletion of the IL-17 gene (54). Our data now demonstrate that deficiency of either CD39 or CD73 leads to polarization toward $\mathrm{T}_{\mathrm{H}} 17$ cells, which we hypothesize to be at least partially attributable to a defect in immunosuppressive Treg function in ectonucleotidase-deficient mice (15).

While this study focused on ectonucleotidases, follow-up studies should further characterize the purine species that they regulate and downstream signaling events. While the conversion of ATP to AMP can be countered by extracellular kinase activity, the conversion of AMP to adenosine can only be reversed upon intracellular transport of adenosine. This places CD73 at a crucial checkpoint in the conversion of extracellular, proinflammatory ATP into anti-inflammatory adenosine. Indeed, in animal models, CD73 has been shown to protect against LPS-induced neutrophil trafficking into lungs (55), and permeability of hypoxic endothelium to neutrophils (12-14). In our hands, CD73 deficiency, when compared with CD39 deficiency, was the greater potentiator of both autoimmune-exacerbating and inflammatory neutrophil phenotypes in the pristane model, thereby hinting that adenosine (and its downstream signaling pathways) in particular warrants further study.

It should be emphasized that our work is performed in a mouse model of lupus and has potential limitations when extrapolated to human lupus. For example, a series of families identified with CD73 null mutations did not have a clinical autoimmune phenotype, but were instead predisposed to severe calcification of lower extremity arteries and joint capsules (56). To the best of our knowledge, CD73 mutations have never been described in lupus patients. There is growing interest in the role of purinergic signaling in immune function, and as a therapeutic target. Recent 
work has described CD38/CD203a as an alternative mechanism of ATP catabolism by both human T cells (perhaps especially Tregs) and cancer cells $(57,58)$. Indeed, antagonism of CD38 is currently being explored as a therapeutic approach to boost the anticancer immune response $(59,60)$. Interestingly, in mice, CD38 deficiency has in some cases been shown to attenuate autoimmune/inflammatory disease (61), again emphasizing differences between these pathways in mouse and human. Now that our study has highlighted the potential importance of purinergic signaling in lupus, future studies should consider parallel/compensatory pathways such as CD38/CD203a, as well as adenosine deaminase $(62,63)$, in both mice and humans.

Adenosine receptors vary in both affinities for adenosine and tissue distribution (10). For example, neutrophils express all four adenosine receptors (64). The $\mathrm{A}_{1}$ receptor (which has a high affinity for adenosine) promotes neutrophil chemotaxis, while the other three receptors (which may only become activated when the environment is flooded with excess adenosine) tend to silence neutrophils $(10,64)$. In particular, the $A_{2 A}$ and $A_{3}$ receptors are expressed at high levels on neutrophils and are recognized as suppressors of neutrophil effector functions (64-66). One study demonstrated that agonism of the $\mathrm{A}_{2 \mathrm{~A}}$ receptor is protective against nephritis in a different model of lupus (35). However, the impact of adenosine signaling on lupus vascular disease and neutrophil activity, and the role of adenosine receptors beyond the $\mathrm{A}_{2 \mathrm{~A}}$ receptor, are heretofore unexplored. Our data now for the first time link ectonucleotidases to the release of NETs by neutrophils. At baseline (i.e., the saline condition) both $\mathrm{CD} 39^{-/-}$ and $\mathrm{CD}_{73^{-/-}}$mice demonstrated exaggerated NET release, which was further potentiated by pristane administration. We speculate that ectonucleotidases play a role in generating a local "halo" of adenosine that suppresses NET release.

In the 1950s, mortality attributable to lupus was more than $50 \%$, with that startling number driven especially by renal failure. With advances in immunosuppressive therapy and transplant medicine, nephritis is now a rare cause of death. In its place, cardiovascular disease has emerged as a leading cause of mortality in lupus (27). While NETs were originally described as key players in host defense, recent work has pointed to a multifaceted (and generally deleterious) intersection with the vasculature. Proteases in NETs kill endothelial cells. NETs stimulate interferon production, which reduces the numbers and function of restorative endothelial progenitors $(21,67)$. Furthermore, in lupus-relevant

\section{REFERENCES}

1. Manzi S, Selzer F, Sutton-Tyrrell K, Fitzgerald SG, Rairie JE, Tracy RP, et al. Prevalence and risk factors of carotid plaque in women with systemic lupus erythematosus. Arthritis Rheum (1999) 42:51-60. doi:10.1002/1529-0131 (199901)42:1<51::AID-ANR7>3.0.CO;2-D

2. Reeves WH, Lee PY, Weinstein JS, Satoh M, Lu L. Induction of autoimmunity by pristane and other naturally occurring hydrocarbons. Trends Immunol (2009) 30:455-64. doi:10.1016/j.it.2009.06.003

3. Zhuang H, Szeto C, Han S, Yang L, Reeves WH. Animal models of interferon signature positive lupus. Front Immunol (2015) 6:291. doi:10.3389/ fimmu.2015.00291

4. Nacionales DC, Kelly-Scumpia KM, Lee PY, Weinstein JS, Lyons R, Sobel E, et al. Deficiency of the type I interferon receptor protects mice from experimental lupus. Arthritis Rheum (2007) 56:3770-83. doi:10.1002/art.23023 mouse models, inhibition of NETosis mitigates both arterial and venous thrombosis $(21,34,68)$. Here, we posit that neutrophil hyperactivity (in the absence of nucleotide dissipation) is an important mediator of the pristane-dependent endothelial dysfunction observed in ectonucleotidase-deficient mice, although confirmation of this will require further study.

In summary, we have revealed a previously unrecognized role for ectonucleotidases in protection against lupus. In particular, these data are the first to link ectonucleotidases with lupus autoimmunity and vascular disease. Future therapeutic strategies may harness purinergic signaling to limit the damage inflicted by lupus upon organs and blood vessels.

\section{ETHICS STATEMENT}

This study was carried out in accordance with the recommendations of the National Research Council, Guide for the Care and Use of Laboratory Animals. The protocol was approved by the Institutional Animal Care and Use Committee.

\section{AUTHOR CONTRIBUTIONS}

LM, SY, GS, RA, JH, and YK conducted experiments and analyzed data. JK, YK, and DP designed the study. All the authors participated in writing the manuscript and gave approval before submission.

\section{ACKNOWLEDGMENTS}

We thank Dr. Wenpu Zhao and Mr. Robert Grenn for their excellent technical assistance. The work was supported by NIH R01HL134846 to JK. JK was also supported by career development awards from the NIH (K08AR066569), Burroughs Wellcome Fund, Arthritis National Research Foundation, and Rheumatology Research Foundation. YK was supported by NIH K08HL131993 and L30HL129373. The work was also partially supported by R01HL127151 and R01NS087147 to DP.

\section{SUPPLEMENTARY MATERIAL}

The Supplementary Material for this article can be found online at https:/www.frontiersin.org/articles/10.3389/fimmu.2018.01322/ full\#supplementary-material.

5. Lee PY, Kumagai Y, Li Y, Takeuchi O, Yoshida H, Weinstein J, et al. TLR7dependent and FcgammaR-independent production of type I interferon in experimental mouse lupus. J Exp Med (2008) 205:2995-3006. doi:10.1084/ jem. 20080462

6. Lourenco EV, Liu A, Matarese G, La Cava A. Leptin promotes systemic lupus erythematosus by increasing autoantibody production and inhibiting immune regulation. Proc Natl Acad Sci U S A (2016) 113:10637-42. doi:10.1073/pnas. 1607101113

7. Wang H, Knight JS, Hodgin JB, Wang J, Guo C, Kleiman K, et al. Psgl-1 deficiency is protective against stroke in a murine model of lupus. Sci Rep (2016) 6:28997. doi:10.1038/srep28997

8. Kahlenberg JM, Yalavarthi S, Zhao W, Hodgin JB, Reed TJ, Tsuji NM, et al. An essential role of caspase 1 in the induction of murine lupus and its associated vascular damage. Arthritis Rheumatol (2014) 66:152-62. doi:10.1002/ art.38225 
9. Salmi M, Jalkanen S. Cell-surface enzymes in control of leukocyte trafficking. Nat Rev Immunol (2005) 5:760-71. doi:10.1038/nri1705

10. Cronstein BN, Sitkovsky M. Adenosine and adenosine receptors in the pathogenesis and treatment of rheumatic diseases. Nat Rev Rheumatol (2017) 13:41-51. doi:10.1038/nrrheum.2016.178

11. Antonioli L, Pacher P, Vizi ES, Hasko G. CD39 and CD73 in immunity and inflammation. Trends Mol Med (2013) 19:355-67. doi:10.1016/j. molmed.2013.03.005

12. Eltzschig HK, Ibla JC, Furuta GT, Leonard MO, Jacobson KA, Enjyoji K, et al. Coordinated adenine nucleotide phosphohydrolysis and nucleoside signaling in posthypoxic endothelium: role of ectonucleotidases and adenosine A2B receptors. J Exp Med (2003) 198:783-96. doi:10.1084/jem.20030891

13. Eltzschig HK, Thompson LF, Karhausen J, Cotta RJ, Ibla JC, Robson SC, et al. Endogenous adenosine produced during hypoxia attenuates neutrophil accumulation: coordination by extracellular nucleotide metabolism. Blood (2004) 104:3986-92. doi:10.1182/blood-2004-06-2066

14. Thompson LF, Eltzschig HK, Ibla JC, Van De Wiele CJ, Resta R, MoroteGarcia JC, et al. Crucial role for ecto-5'-nucleotidase (CD73) in vascular leakage during hypoxia. J Exp Med (2004) 200:1395-405. doi:10.1084/jem. 20040915

15. Deaglio S, Dwyer KM, Gao W, Friedman D, Usheva A, Erat A, et al. Adenosine generation catalyzed by $\mathrm{CD} 39$ and $\mathrm{CD} 73$ expressed on regulatory $\mathrm{T}$ cells mediates immune suppression. J Exp Med (2007) 204:1257-65. doi:10.1084/ jem.20062512

16. Ryzhov SV, Pickup MW, Chytil A, Gorska AE, Zhang Q, Owens P, et al. Role of TGF-beta signaling in generation of CD39+CD73+ myeloid cells in tumors. J Immunol (2014) 193:3155-64. doi:10.4049/jimmunol.1400578

17. Li DM, Li XP, Zhang JH, Hu SR, Xiao B, Chen W, et al. [The expression of CD73 in CD4+ regulatory $\mathrm{T}$ cells in patients with new-onset systemic lupus erythematosus]. Zhonghua Nei Ke Za Zhi (2010) 49:772-5.

18. Loza MJ, Anderson AS, O'Rourke KS, Wood J, Khan IU. T-cell specific defect in expression of the NTPDase CD39 as a biomarker for lupus. Cell Immunol (2011) 271:110-7. doi:10.1016/j.cellimm.2011.06.010

19. Visovatti SH, Hyman MC, Goonewardena SN, Anyanwu AC, Kanthi Y, Robichaud P, et al. Purinergic dysregulation in pulmonary hypertension. Am J Physiol Heart Circ Physiol (2016) 311:H286-98. doi:10.1152/ ajpheart.00572.2015

20. Petrovic-Djergovic D, Hyman MC, Ray JJ, Bouis D, Visovatti SH, Hayasaki T, et al. Tissue-resident ecto-5' nucleotidase (CD73) regulates leukocyte trafficking in the ischemic brain. J Immunol (2012) 188:2387-98. doi:10.4049/ jimmunol.1003671

21. Knight JS, Zhao W, Luo W, Subramanian V, O’Dell AA, Yalavarthi S, et al. Peptidylarginine deiminase inhibition is immunomodulatory and vasculoprotective in murine lupus. J Clin Invest (2013) 123:2981-93. doi:10.1172/ JCI67390

22. Knight JS, Subramanian V, O'Dell AA, Yalavarthi S, Zhao W, Smith CK, et al. Peptidylarginine deiminase inhibition disrupts NET formation and protects against kidney, skin and vascular disease in lupus-prone MRL/lpr mice. Ann Rheum Dis (2015) 74:2199-206. doi:10.1136/annrheumdis-2014-205365

23. Whitesall SE, Hoff JB, Vollmer AP, D’Alecy LG. Comparison of simultaneous measurement of mouse systolic arterial blood pressure by radiotelemetry and tail-cuff methods. Am J Physiol Heart Circ Physiol (2004) 286:H2408-15. doi:10.1152/ajpheart.01089.2003

24. Barker TT, Lee PY, Kelly-Scumpia KM, Weinstein JS, Nacionales DC, Kumagai Y, et al. Pathogenic role of B cells in the development of diffuse alveolar hemorrhage induced by pristane. Lab Invest (2011) 91:1540-50. doi:10.1038/labinvest.2011.108

25. Zhuang H, Han S, Lee PY, Khaybullin R, Shumyak S, Lu L, et al. Pathogenesis of diffuse alveolar hemorrhage in murine lupus. Arthritis Rheumatol (2017) 69(6):1280-93. doi:10.1002/art.40077

26. Chowdhary VR, Grande JP, Luthra HS, David CS. Characterization of haemorrhagic pulmonary capillaritis: another manifestation of pristane-induced lupus. Rheumatology (Oxford) (2007) 46:1405-10. doi:10.1093/rheumatology/ kem117

27. Knight JS, Kaplan MJ. Cardiovascular disease in lupus: insights and updates. CurrOpinRheumatol(2013)25:597-605.doi:10.1097/BOR.0b013e328363eba3

28. Knight JS, Kaplan MJ. Lupus neutrophils: 'NET' gain in understanding lupus pathogenesis. Curr Opin Rheumatol (2012) 24:441-50. doi:10.1097/ BOR.0b013e3283546703
29. Grayson PC, Kaplan MJ. At the bench: neutrophil extracellular traps (NETs) highlight novel aspects of innate immune system involvement in autoimmune diseases. J Leukoc Biol (2016) 99:253-64. doi:10.1189/jlb.5BT0615-247R

30. Banchereau R, Hong S, Cantarel B, Baldwin N, Baisch J, Edens M, et al. Personalized immunomonitoring uncovers molecular networks that stratify lupus patients. Cell (2016) 165:551-65. doi:10.1016/j.cell.2016.03.008

31. Jourde-Chiche N, Whalen E, Gondouin B, Speake C, Gersuk V, Dussol B, et al. Modular transcriptional repertoire analyses identify a blood neutrophil signature as a candidate biomarker for lupus nephritis. Rheumatology (Oxford) (2017) 56:477-87. doi:10.1093/rheumatology/kew439

32. Caielli S, Athale S, Domic B, Murat E, Chandra M, Banchereau R, et al. Oxidized mitochondrial nucleoids released by neutrophils drive type I interferon production in human lupus. J Exp Med (2016) 213:697-713. doi:10.1084/jem.20151876

33. Lood C, Blanco LP, Purmalek MM, Carmona-Rivera C, De Ravin SS, Smith CK, et al. Neutrophil extracellular traps enriched in oxidized mitochondrial DNA are interferogenic and contribute to lupus-like disease. Nat Med (2016) 22:146-53. doi:10.1038/nm.4027

34. Knight JS, Luo W, O’Dell AA, Yalavarthi S, Zhao W, Subramanian V, et al. Peptidylarginine deiminase inhibition reduces vascular damage and modulates innate immune responses in murine models of atherosclerosis. Circ Res (2014) 114:947-56. doi:10.1161/CIRCRESAHA.114.303312

35. Zhang L, Yang N, Wang S, Huang B, Li F, Tan H, et al. Adenosine 2A receptor is protective against renal injury in MRL/lpr mice. Lupus (2011) 20:667-77. doi:10.1177/0961203310393262

36. Stancikova M, Lukac J, Istok R, Cristalli G, Rovensky J. Serum adenosine deaminase activity and its isoenzyme pattern in patients with systemic lupus erythematosus. Clin Exp Rheumatol (1998) 16:583-6.

37. Saghiri R, Ghashghai N, Movaseghi S, Poursharifi P, Jalilfar S, Bidhendi MA, et al. Serum adenosine deaminase activity in patients with systemic lupus erythematosus: a study based on $\mathrm{ADA} 1$ and $\mathrm{ADA} 2$ isoenzymes pattern. Rheumatol Int (2012) 32:1633-8. doi:10.1007/s00296-011-1836-8

38. Bortoluzzi A, Vincenzi F, Govoni M, Padovan M, Ravani A, Borea PA, et al. A2A adenosine receptor upregulation correlates with disease activity in patients with systemic lupus erythematosus. Arthritis Res Ther (2016) 18:192. doi:10.1186/s13075-016-1089-8

39. Blume C, Felix A, Shushakova N, Gueler F, Falk CS, Haller H, et al. Autoimmunity in CD73/Ecto-5'-nucleotidase deficient mice induces renal injury. PLoS One (2012) 7:e37100. doi:10.1371/journal.pone.0037100

40. Dong K, Gao ZW, Zhang HZ. The role of adenosinergic pathway in human autoimmune diseases. Immunol Res (2016) 64:1133-41. doi:10.1007/s12026016-8870-2

41. Chrobak P, Charlebois R, Rejtar P, El Bikai R, Allard B, Stagg J. CD73 plays a protective role in collagen-induced arthritis. J Immunol (2015) 194:2487-92. doi:10.4049/jimmunol.1401416

42. Peres RS, Liew FY, Talbot J, Carregaro V, Oliveira RD, Almeida SL, et al. Low expression of $\mathrm{CD} 39$ on regulatory $\mathrm{T}$ cells as a biomarker for resistance to methotrexate therapy in rheumatoid arthritis. Proc Natl Acad Sci U S A (2015) 112:2509-14. doi:10.1073/pnas.1424792112

43. Botta Gordon-Smith S, Ursu S, Eaton S, Moncrieffe H, Wedderburn LR. Correlation of low CD73 expression on synovial lymphocytes with reduced adenosine generation and higher disease severity in juvenile idiopathic arthritis. Arthritis Rheumatol (2015) 67:545-54. doi:10.1002/art.38959

44. Friedman DJ, Kunzli BM, Yi AR, Sevigny J, Berberat PO, Enjyoji K, et al. From the cover: $\mathrm{CD} 39$ deletion exacerbates experimental murine colitis and human polymorphisms increase susceptibility to inflammatory bowel disease. Proc Natl Acad Sci U S A (2009) 106:16788-93. doi:10.1073/pnas.0902869106

45. Bynoe MS, Waickman AT, Mahamed DA, Mueller C, Mills JH, Czopik A. $\mathrm{CD} 73$ is critical for the resolution of murine colonic inflammation. J Biomed Biotechnol (2012) 2012:260983. doi:10.1155/2012/260983

46. Grant CR, Liberal R, Holder BS, Cardone J, Ma Y, Robson SC, et al. Dysfunctional CD39(POS) regulatory T cells and aberrant control of T-helper type 17 cells in autoimmune hepatitis. Hepatology (2014) 59:1007-15. doi:10.1002/hep.26583

47. Buchheiser A, Ebner A, Burghoff S, Ding Z, Romio M, Viethen C, et al. Inactivation of CD73 promotes atherogenesis in apolipoprotein E-deficient mice. Cardiovasc Res (2011) 92:338-47. doi:10.1093/cvr/cvr218

48. Kanthi Y, Hyman MC, Liao H, Baek AE, Visovatti SH, Sutton NR, et al. Flow-dependent expression of ectonucleotide tri(di)phosphohydrolase-1 and suppression of atherosclerosis. J Clin Invest (2015) 125:3027-36. doi:10.1172/ JCI79514 
49. Kobie JJ, Shah PR, Yang L, Rebhahn JA, Fowell DJ, Mosmann TR. T regulatory and primed uncommitted $\mathrm{CD} 4 \mathrm{~T}$ cells express CD73, which suppresses effector CD4 T cells by converting 5'-adenosine monophosphate to adenosine. J Immunol (2006) 177:6780-6. doi:10.4049/jimmunol.177.10.6780

50. Zarek PE, Huang CT, Lutz ER, Kowalski J, Horton MR, Linden J, et al. A2A receptor signaling promotes peripheral tolerance by inducing T-cell anergy and the generation of adaptive regulatory T cells. Blood (2008) 111:251-9. doi:10.1182/blood-2007-03-081646

51. Zhang Z, Kyttaris VC, Tsokos GC. The role of IL-23/IL-17 axis in lupus nephritis. J Immunol (2009) 183:3160-9. doi:10.4049/jimmunol.0900385

52. Chen XQ, Yu YC, Deng HH, Sun JZ, Dai Z, Wu YW, et al. Plasma IL-17A is increased in new-onset SLE patients and associated with disease activity. J Clin Immunol (2010) 30:221-5. doi:10.1007/s10875-009-9365-x

53. Shah K, Lee WW, Lee SH, Kim SH, Kang SW, Craft J, et al. Dysregulated balance of Th17 and Th1 cells in systemic lupus erythematosus. Arthritis Res Ther (2010) 12:R53. doi:10.1186/ar2964

54. Amarilyo G, Lourenco EV, Shi FD, La Cava A. IL-17 promotes murine lupus. J Immunol (2014) 193:540-3. doi:10.4049/jimmunol.1400931

55. Reutershan J, Vollmer I, Stark S, Wagner R, Ngamsri KC, Eltzschig HK. Adenosine and inflammation: CD39 and CD73 are critical mediators in LPSinduced PMN trafficking into the lungs. FASEB J (2009) 23:473-82. doi:10.1096/ f. $08-119701$

56. St Hilaire C, Ziegler SG, Markello TC, Brusco A, Groden C, Gill F, et al. NT5E mutations and arterial calcifications. N Engl J Med (2011) 364:432-42. doi:10.1056/NEJMoa0912923

57. Horenstein AL, Chillemi A, Zaccarello G, Bruzzone S, Quarona V, Zito A, et al. A CD38/CD203a/CD73 ectoenzymatic pathway independent of CD39 drives a novel adenosinergic loop in human T lymphocytes. Oncoimmunology (2013) 2:e26246. doi:10.4161/onci.26246

58. Morandi F, Morandi B, Horenstein AL, Chillemi A, Quarona V, Zaccarello G, et al. A non-canonical adenosinergic pathway led by CD38 in human melanoma cells induces suppression of T cell proliferation. Oncotarget (2015) 6:25602-18. doi:10.18632/oncotarget.4693

59. Krejcik J, Casneuf T, Nijhof IS, Verbist B, Bald J, Plesner T, et al. Daratumumab depletes $\mathrm{CD} 38+$ immune regulatory cells, promotes $\mathrm{T}$-cell expansion, and skews T-cell repertoire in multiple myeloma. Blood (2016) 128:384-94. doi:10.1182/blood-2015-12-687749

60. Feng X, Zhang L, Acharya C, An G, Wen K, Qiu L, et al. Targeting CD38 suppresses induction and function of $\mathrm{T}$ regulatory cells to mitigate immunosuppression in multiple myeloma. Clin Cancer Res (2017) 23:4290-300. doi:10.1158/1078-0432.CCR-16-3192
61. Postigo J, Iglesias M, Cerezo-Wallis D, Rosal-Vela A, Garcia-Rodriguez S, Zubiaur M, et al. Mice deficient in CD38 develop an attenuated form of collagen type II-induced arthritis. PLoS One (2012) 7:e33534. doi:10.1371/ journal.pone. 0033534

62. Sauer AV, Brigida I, Carriglio N, Hernandez RJ, Scaramuzza S, Clavenna D, et al. Alterations in the adenosine metabolism and CD39/CD73 adenosinergic machinery cause loss of Treg cell function and autoimmunity in ADA-deficient SCID. Blood (2012) 119:1428-39. doi:10.1182/blood-2011-07-366781

63. Gao ZW, Zhao GH, Zhang Z, Huang J, Li ZY, Zhang HZ, et al. Serum adenosine deaminase activity is increased in systemic lupus erythematosus patients and correlated with disease activity. Immunol Res (2018) 66(2):299-304. doi:10.1007/s12026-018-8984-9

64. Barletta KE, Ley K, Mehrad B. Regulation of neutrophil function by adenosine. Arterioscler Thromb Vasc Biol (2012) 32:856-64. doi:10.1161/ATVBAHA. 111.226845

65. Cronstein BN, Kramer SB, Weissmann G, Hirschhorn R. Adenosine: a physiological modulator of superoxide anion generation by human neutrophils. J Exp Med (1983) 158:1160-77. doi:10.1084/jem.158.4.1160

66. van der Hoeven D, Wan TC, Auchampach JA. Activation of the A(3) adenosine receptor suppresses superoxide production and chemotaxis of mouse bone marrow neutrophils. Mol Pharmacol (2008) 74:685-96. doi:10.1124/ mol.108.048066

67. Grenn RC, Yalavarthi S, Gandhi AA, Kazzaz NM, Nunez-Alvarez C, Hernandez-Ramirez D, et al. Endothelial progenitor dysfunction associates with a type I interferon signature in primary antiphospholipid syndrome. Ann Rheum Dis (2017) 76:450-7. doi:10.1136/annrheumdis-2016-209442

68. Meng H, Yalavarthi S, Kanthi Y, Mazza LF, Elfline MA, Luke CE, et al. In vivo role of neutrophil extracellular traps in antiphospholipid antibody-mediated venous thrombosis. Arthritis Rheumatol (2017) 69(3):655-67. doi:10.1002/ art.39938

Conflict of Interest Statement: The authors declare that the research was conducted in the absence of any commercial or financial relationships that could be construed as a potential conflict of interest.

Copyright $(2018$ Knight, Mazza, Yalavarthi, Sule, Ali, Hodgin, Kanthi and Pinsky. This is an open-access article distributed under the terms of the Creative Commons Attribution License (CC BY). The use, distribution or reproduction in other forums is permitted, provided the original author(s) and the copyright owner are credited and that the original publication in this journal is cited, in accordance with accepted academic practice. No use, distribution or reproduction is permitted which does not comply with these terms. 